\title{
Current Procedural Terminology Codes for Medication Therapy Management in Administrative Data
}

\author{
David R. Axon, PhD, MPharm, MS; Chanadda Chinthammit, BPharm, MS, PhD; \\ Jared Tate, PharmD; Ann M. Taylor, MPH, MCHES; Sandra Leal, PharmD, MPH, FAPhA; \\ Matthew Pickering, PharmD; Heather Black, PhD; Terri Warholak, PhD, RPh, FAPhA; \\ and Patrick J. Campbell, PhD, PharmD, RPh
}

\begin{abstract}
BACKGROUND: Three pharmacist-specific Current Procedural Terminology (CPT) codes exist to facilitate medication therapy management (MTM) reimbursement (codes 99605, 99606, and 99607). However, no studies have used CPT codes in administrative claims databases to identify subjects who have received MTM services.

OBJECTIVE: To assess the prevalence of MTM services provided, using CPT codes identified in an administrative dataset.

METHODS: A retrospective cohort study was conducted using a subset of Medicare Part D individuals from the IBM MarketScan Medicare Supplemental Research Databases (2009-2015). Researchers identified beneficiaries who received MTM services using CPT codes 99605, 99606, and 99607.

RESULTS: Of the $16,483,709$ individuals in the dataset, only 3,291 had CPT codes indicating that they received MTM services, representing an overall prevalence of $0.020 \%$.

CONCLUSIONS: The use of CPT codes as an indicator of MTM service provision resulted in far lower MTM utilization rates than in published literature. Reliance on CPT codes to identify MTM services in administrative claims is not recommended, given that it limited the researchers' ability to properly identify patient receipt of such services. More accurate methodologies are warranted for identifying MTM use and its effects on patient outcomes.
\end{abstract}

J Manag Care Spec Pharm. 2020;26(10):1297-300

Copyright $\odot 2020$, Academy of Managed Care Pharmacy. All rights reserved.

\section{What is already known about this subject}

- Three pharmacist-specific Current Procedural Terminology (CPT) codes exist to facilitate medication therapy management (MTM) reimbursement (codes 99605, 99606, and 99607).

Large databases that contain these codes provide an opportunity to assess the effectiveness of MTM services.

However, no studies have used CPT codes in administrative claims databases to identify subjects who have received MTM services.

\section{What this study adds}

This retrospective cohort study found only 3,291 of 16,483,709 (0.020\%) Medicare Part D individuals in the IBM MarketScan Medicare Supplemental Research Databases (2009-2015) had CPT)codes 99605, 99606, and 99607, indicating that they received MTM services.

- It was not possible to identify all patients in receipt of MTM services, thus, use of CPT codes to identify MTM services in administrative claims is not recommended.

Identification and evaluation of more accurate methodologies representing MTM services are needed.

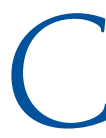

hronic disease management, especially for those with numerous conditions taking multiple medications, presents an ongoing challenge for patients and health care providers. To address this challenge, the Medicare Modernization Act of 2003, overseen by the Centers for Medicare \& Medicaid Services (CMS), introduced medication therapy management (MTM) and required its provision for all eligible Medicare Part D beneficiaries. ${ }^{1}$ The American Pharmacists Association (APhA) defines MTM as "a service or group of services that optimize therapeutic outcomes for individual patients." ${ }^{2}$ Despite various delivery methods (i.e., telephonic, face-to-face, or both), ${ }^{3}$ APhA delineated 5 core elements to describe the services: (1) medication therapy review, commonly referred to as a comprehensive medication review (CMR); (2) personal medication record; (3) medication-related action plan; (4) intervention and/or referral; and (5) documentation and follow-up. ${ }^{2}$

CMS determines eligibility for MTM services using an opt-in approach, whereby beneficiaries who choose not to participate must verbally opt out. Eligible Medicare Part D beneficiaries must (a) have multiple chronic conditions (minimum of 2 or 3 at Medicare Part D sponsor's discretion); (b) take multiple chronic medications; and (c) have a projected drug spend above a predetermined annual cost threshold for Medicare Part D medications ( $\$ 3,919$ in 2017) - this maximum is adjusted annually by CMS. ${ }^{3}$ 
Since its inception, growing evidence supports MTM's positive clinical and economic benefits. ${ }^{4-9}$ Studies have also reported how virtual telehealth programs were able to provide MTM services to patients without in-person access to a pharmacist or other health care providers. ${ }^{10}$ In 1 study, MTM services resulted in over 200 medication-related interventions and over 1,100 health promotion interventions among 517 rural-dwelling patients with diabetes and/or hypertension. ${ }^{11}$ In another example, over 300 recommendations were made by an MTM pharmacist to 86 people with epilepsy. ${ }^{12}$ However, several challenges remain that limit the effectiveness of the respective interventions. ${ }^{13}$ For example, less than half (24\%$50 \%$ ) of eligible beneficiaries complete a CMR thus limiting the scope and ability to measure MTM's effectiveness in improving patient-related outcomes. ${ }^{14}$ To better evaluate rates, CMS adopted the performance measure for CMR completion rates endorsed by the Pharmacy Quality Alliance (PQA) as part of the Medicare Part D star ratings, which can be used to distinguish high- versus low-quality performing sponsors. ${ }^{15}$ The PQA CMR measure assesses the percentage of prescription drug plan members who are eligible for MTM and who received a CMR during the eligibility period.

This focused attention on MTM, coupled with the potential for improving clinical and economic outcomes via the star ratings (e.g., triple-weighted adherence measures) have prompted sponsors to improve, innovate, and evaluate the provision of their services. Specifically, MTM's effectiveness has been evaluated prospectively and retrospectively with demonstrated reductions in hemoglobin Alc; lipid levels and mean direct medical costs ${ }^{4}$; improved Healthcare Effectiveness Data and Information Set (HEDIS) measures ${ }^{6}$; and decreased health care expenditures. ${ }^{6,9}$ As such, some of the largest retrospective reviews have used data obtained directly from MTM vendors or health plans.

Administrative database studies, an approach absent in the MTM literature to date, has several potential advantages, including larger sample size; access to relevant costs information (e.g., related to medication, nonmedication, and total expenditures); health care utilization information (e.g., emergency department visits, hospitalization, and outpatient visits); and clinical information (e.g., measures of medication adherence via proportion of days covered data and laboratory values). Furthermore, pharmacists are the most frequent providers of MTM services ${ }^{3}$; therefore, CMS created 3 pharmacistspecific Current Procedural Terminology (CPT) codes to facilitate MTM reimbursement: code 99605-first-time MTM consultation (up to 15 minutes); code 99606-follow-up MTM consultation (up to 15 minutes); and code 99607-additional 15 minutes of an existing consultation for use in tandem with an initial (99605) or follow-up (99606) MTM consultation.

\begin{tabular}{l|rr}
\hline TABLE 1) & \multicolumn{2}{c}{$\begin{array}{l}\text { Number of Individuals with CPT Codes } \\
\text { by Year, 2009-2015 ( } \mathbf{N}=16,483,709)\end{array}$} \\
\hline Year & \multicolumn{2}{|c}{$\mathbf{N}(\%)$} \\
\hline 2009 & 113 & $(0.003)$ \\
\hline 2010 & 233 & $(0.005)$ \\
\hline 2011 & 267 & $(0.010)$ \\
\hline 2012 & 304 & $(0.012)$ \\
\hline 2013 & 715 & $(0.034)$ \\
\hline 2014 & 1,586 & $(0.098)$ \\
\hline 2015 & 3,291 & $(0.020)$ \\
\hline Total & \multicolumn{2}{l}{} \\
\hline CPT=Current Procedural Terminology.
\end{tabular}

While code 99607 must be used with codes 99605 or 99606 , it provides opportunities for further reimbursement because of the longer consultation times. Using large databases that contain these specific codes and the potential advantages of using them over other methods provides an excellent opportunity to conduct further research to assess the effectiveness of MTM services. However, it is critical to initially assess the validity of using CPT codes as a method to identify MTM services in administrative datasets. Furthermore, to date, no studies have used CPT codes in administrative claims to identify subjects that have received MTM services.

The objective of this study was to describe the feasibility of identifying MTM services via an administrative claims database, using pharmacist-specific CPT codes used for MTM billing.

\section{Methods}

This retrospective cohort study used a subset of the IBM MarketScan Medicare Supplemental Research Databases (2009-2015). The number of unique individuals in the dataset was calculated using data from the enrollment file. Subjects with any enrollment data were included. Researchers identified subjects with CPT codes 99605, 99606, and/or 99607 listed at any time between 2009 and 2015 from the inpatient and outpatient services files. The unique number of subjects with at least 1 MTM CPT code was calculated. The prevalence of individuals receiving MTM was estimated overall and by calendar year. The University of Arizona Institutional Review Board approved this study.

\section{Results}

A total of 16,483,709 individuals were identified in the dataset, with 3,291 of these having at least 1 of the 3 MTM CPT codes. From 2009 to 2015, the overall MTM prevalence estimate was $0.020 \%$. The number and percentage of individuals with CPT codes for each year in the study is shown in Table 1. Annual MTM prevalence estimates ranged from 0.003\% ( $n=73)$ in 2009 and increased year after year to $0.098 \%(n=1,586)$ in 2015 . 


\section{Discussion}

This is the first study to describe the feasibility of using CPT codes to identify MTM services in administrative claims. Interestingly, very few individuals were identified with MTMrelated CPT codes in the Medicare supplemental dataset, contrary to actual MTM use among Medicare beneficiaries. For instance, 2017 CMS star measure data (based on plan performance reported for 2015) indicates a 40\% national average CMR completion rate, based on a star measure and marker of MTM services, ${ }^{16}$ while the current findings (0.098\%) represent an overall prevalence. However, this still represents a 400-fold difference between the 2 study findings, which strongly suggests that using MTM-related CPT codes may not be an appropriate method for tracking patient identification and MTM service use.

While other CPT codes for "Medication Reconciliation PostDischarge" and "Non-Face-to-Face Non-physician Telephonic Services" (1111F and 98966, respectively) are used to identify service provision, it seemed reasonable to assume that selecting MTM-specific CPT codes for tracking receipt of services would net similar results. Yet, this approach had major limitations, as described below, and is not recommended.

\section{Limitations}

This study had limitations that need to be considered. First, a large proportion of MTM services are provided by covered entities. While CMS mandates provision of these services for eligible beneficiaries, it does not specifically delineate the actual process whereby the delivery occurs. Medicare Part D sponsors are afforded latitude in subcontracting these services to outside covered entities such as pharmacy benefit managers (PBMs), MTM vendors, community pharmacists, or long-term care pharmacists. Currently, $92.9 \%$ of programs contract out for these services via external entities that include PBMs (56.0\%) and MTM vendors (84.7\%). ${ }^{3}$ It is noteworthy that these are not mutually exclusive categories, given that PBMs often use an MTM vendor, separate from their relationship with the Medicare Part D sponsor. In this type of arrangement, contracting often uses payment structures that bill on a perqualified-member or per-member per-month basis or a flat rate for services provided. Thus, a large proportion of MTM service provision would remain uncaptured if CPT codes were used as a marker of delivery. Furthermore, in the current study, CPT codes were identified from a Medicare supplemental dataset, so it is possible that these individuals received MTM services yet were billed elsewhere.

Second, while physicians and other nonpharmacist providers may perform MTM services, the available CPT codes are currently exclusive for pharmacists and pharmacy services. Therefore, use of these codes would not capture the expanse of MTM services provided by other health care providers, who may use CPT codes that they are already familiar with, such as those for established (99201-99205) and newly established (99211-99215) patients. In addition, when these MTM codes were created, no associated fee schedule existed. In the interim, payers were required to establish their own fee schedules, yet many failed to embrace this and subsequently did not do so. As such, pharmacists continued to work within the established codes to generate revenue, although they were providing MTM services. Pharmacists also cannot bill using the usual codes that physicians and other nonphysician providers use, since they are not recognized as providers by CMS so must bill under a Medicare-credentialed provider, a common practice referred to as "incident-to" billing. ${ }^{17}$ Historically, pharmacists have used "incident-to" billing for provision of MTM services, which resulted in the inability to accurately track and report service provision, often resulting in devaluation of their services.

Finally, and most importantly, MTM codes were not exclusively used for Medicare Part D billing but were designed with the intent of using the respective codes (i.e., 99605, 99606, and 99607) in any situation where the services were provided. However, MTM in Medicare Part D has specific qualifying criteria, yet pharmacists using these codes in Medicare Part B, commercial, or Medicaid plans do not necessarily use those same criteria to provide the service. ${ }^{18}$

In the future, we suggest that researchers could consider using Systematized Nomenclature of Medicine-Clinical Terms (SNOMED CT) codes to identify MTM services provided. The new Enhanced MTM (EMTM) model requires the use of SNOMED CT codes to capture services provided, so they should become more prevalent in the future. For some organizations, SNOMED CT codes can be easily captured or mapped to existing data, yet for others, there may be financial or other barriers to implementing SNOMED CT codes that need to be resolved. ${ }^{19}$

\section{Conclusions}

This administrative database study, using CPT codes 99605 , 99606, and 99607 as an indicator of MTM service provision, resulted in a far lower prevalence than in the published literature. Various factors may have influenced these, such as subcontracted service delivery, use of inappropriate billing codes, and variation in service provision. Thus, identification and evaluation of more accurate methodologies representing MTM service use are warranted. 


\section{Authors}

DAVID R. AXON, PhD, MPharm, MS; CHANADDA

CHINTHAMMIT, BPharm, MS, PhD; JARED TATE, PharmD;

ANN M. TAYLOR, MPH, MCHES; TERRI WARHOLAK, PhD,

RPh, FAPhA; and PATRICK J. CAMPBELL, PhD, PharmD, RPh,

Department of Pharmacy Practice and Science, University of

Arizona College of Pharmacy, Tucson. SANDRA LEAL, PharmD,

MPH, FAPhA, Tabula Rasa HealthCare, Tucson, Arizona;

MATTHEW PICKERING, PharmD, Quality Measurement Research \& Quality Strategies, Pharmacy Quality Alliance, Alexandria,

Virginia; and HEATHER BLACK, PhD, Outcomes Research, Merck \& Co., Kenilworth, New Jersey.

AUTHOR CORRESPONDENCE: David R. Axon, PhD, MPharm, MS, Assistant Professor, University of Arizona College of Pharmacy, 1295 N. Martin Ave., PO Box 210202, Tucson, AZ, 85721

Tel.: 520.621.961; Email: axon@pharmacy.arizona.edu.

\section{DISCLOSURES}

This work was supported by Pharmacy Quality Alliance; Merck Sharp \& Dohme, a subsidiary of Merck \& Co. (Kenilworth, NJ); and SinfoniaRx. The funding sources had no role in study design, collection, analysis, and interpretation of data, writing the report, or decision to submit the article for publication. Tate, Chinthammit, and Campbell completed this work during their employment at the University of Arizona. Pickering was an employee of Pharmacy Quality Alliance at the time of this study. Black is employed by Merck. Axon reports grants from the Arizona Department of Health Services and the American Association of Colleges of Pharmacy; Campbell reports a grant from the Community Pharmacy Foundation; Chinthammit reports fees from Eli Lilly; Black has received a grant from Merck; Warholak reports grants from the Arizona Department of Health Services and Novartis, all unrelated to this study. Taylor reports grants from Tabula Rasa Op-Co, during the conduct of the study, and from the Arizona Department of Health Services, outside the conduct of this study.

This research was accepted as a poster presentation at the International Society for Pharmacoeconomics and Outcomes Research Annual Meeting, May 16-20, 2020, in Orlando, FL, but was not presented due to the COVID-19 pandemic. An abstract was published in Value in Health, 2020;23(Suppl 1):S305.

\section{REFERENCES}

1. Medicare Prescription Drug, Improvement, and Modernization Act of 2003. 1st ed. 108th Cong. 2004. Available at: https://www.congress.gov/ bill/108th-congress/house-bill/1. Accessed August 15, 2020.

2. American Pharmacists Association, National Association of Chain Drug Stores Foundation. Medication therapy management in pharmacy practice: core elements of an MTM service model (version 2.0). J Am Pharm Assoc (2003). 2008;48(3):341-53.

3. Centers for Medicare \& Medicaid Services. 2017 Medicare Part D medication therapy management (MTM) programs. Fact sheet. August 16, 2017. Available at: https://www.cms.gov/Medicare/Prescription-DrugCoverage/PrescriptionDrugCovContra/Downloads/CY2017-MTM-Fact-Sheet. pdf. Accessed August 15, 2020.
4. Cranor CW, Bunting BA, Christensen DB. The Asheville Project: longterm clinical and economic outcomes of a community pharmacy diabetes care program. J Am Pharm Assoc (Wash). 2003;43(2):173-84.

5. Hurwitz JT, Grizzle AJ, Augustine J, et al. Accepting medication therapy management recommendations to add ACEIs or ARBs in diabetes care. J Manag Care Spec Pharm. 2016;22(1):40-48. Available at: https://www.jmcp. org/doi/10.18553/jmcp.2016.22.1.40.

6. Isetts BJ, Schondelmeyer SW, Artz MB, et al. Clinical and economic outcomes of medication therapy management services: the Minnesota experience. J Am Pharm Assoc (2003). 2008;48(2):203-14.

7. Moczygemba LR, Barner JC, Lawson KA, et al. Impact of telephone medication therapy management on medication and health-related problems, medication adherence, and Medicare Part D drug costs: a 6-month follow up. Am J Geriatr Pharmacother. 2011;9(5):328-38.

8. Ramalho de Oliveira D, Brummel AR, Miller DB. Medication therapy management: 10 years of experience in a large integrated health care system. J Manag Care Pharm. 2010;16(3):185-95. Available at: https://www.jmcp.org/ doi/10.18553/jmcp.2010.16.3.185.

9. Ward MA, Xu Y. Pharmacist-provided telephonic medication therapy management in an MAPD plan. Am J Manag Care. 2011;17(10):e399-409.

10. Taylor AM, Bingham J, Schussel K, et al. Integrated innovative telehealth solutions into an interprofessional team-delivered chronic care management pilot program. J Manag Care Spec Pharm. 2018;24(8):813-18. Available at: https://www.jmcp.org/doi/10.18553/jmcp.2018.24.8.813.

11. Johnson M, Jastrzab R, Tate J, et al. Evaluation of an academiccommunity partnership to implement MTM services in rural communities to improve pharmaceutical care for patients with diabetes and/or hypertension. J Manag Care Spec Pharm. 2018;24(2):132-41. Available at: https://www.jmcp. org/doi/10.18553/jmcp.2018.24.2.132

12. Tetuan C, Axon DR, Bingham J, et al. Assessing the effect of a telepharmacist's recommendations during an integrated, interprofessional telehealth appointment and their alignment with quality measures. J Manag Care Spec Pharm. 2019;25(12):1334-39. Available at: https://www.jmcp.org/ doi/10.18553/jmcp.2019.25.12.1334.

13. Oladapo AO, Rascati KL. Review of survey articles regarding medication therapy management (MTM) services/programs in the United States. J Pharm Prac. 2012;25(4):457-70.

14. Miller DE, Roane TE, Salo JA, Hardin HC. Evaluation of comprehensive medication review completion rates using 3 patient outreach models. J Manag Care Spec Pharm. 2016;22(7):796-800. Available at: https://www. jmcp.org/doi/10.18553/jmcp.2016.22.7.796.

15. Pharmacy Quality Alliance. PQA quality measures. Available at: https:// www.pqaalliance.org/pqa-measures. Accessed August 15, 2020.

16. Centers for Medicare \& Medicaid Services. CMS 2017 star ratings and display measures. Available at: https://www.cms.gov/Medicare/PrescriptionDrug-Coverage/PrescriptionDrugCovGenIn/PerformanceData.html. Accessed August 15, 2020.

17. Verhovshek GJ. The basics of incident-to billing. Physicians Practice. November 23, 2016. Available at: https://www.physicianspractice.com/ medical-billing-collections/basics-incident-billing. Accessed August 15, 2020.

18. Pharmacist Services Technical Advisory Coalition. Frequently asked questions. Available at: http://www.pstac.org/aboutus/faq.html\#_cpt3. Accessed August 15, 2020.

19. Barlas S. CMS to test Enhanced Medication Therapy Management model aims for greater use of pharmacists, cost savings, and better outcomes. P T. 2016;41(7):423-25. 